H. Osada

Nagoya Math. J.

Vol. 113 (1989), 147-151

\title{
NOTE ON THE CLASS-NUMBER OF THE MAXIMAL REAL SUBFIELD OF A GYCLOTOMIC FIELD, II
}

\author{
HIROYUKI OSADA
}

For an integer $m>2$, we denote by $C(m)$ and $H(m)$ the ideal class group and the class-number of the field

$$
K=\boldsymbol{Q}\left(\zeta_{m}+\zeta_{m}^{-1}\right)
$$

respectively, where $\zeta_{m}$ is a primitive $m$-th root of unity. Let $q$ be a prime and $k / \boldsymbol{Q}$ be a real cyclic extension of degree $q$. Let $C(k)$ and $h(k)$ be the ideal class group and the class-number of $k$. In this paper, we give a relation between $C(k)$ (resp. $h(k)$ ) and $C(m)$ (resp. $H(m)$ ) in the case that $m$ is the conductor of $k$ (Main Theorem). As applications of this main theorem, we give the following three propositions. In the previous paper [4], we showed that there exist infinitely many square-free integers $m$ satisfying $n \mid H(m)$ for any given natural number $n$. Using the result of Nakahara [2], we give first an effective sufficient condition for an integer $m$ to satisfy $n \mid H(m)$ for any given natural number $n$ (Proposition 1). Using the result of Nakano [3], we show next that there exist infinitely many positive square-free integers $m$ such that the ideal class group $C(m)$ has a subgroup which is isomorphic to $(Z / n Z)^{2}$ for any given natural number $n$ (Proposition 2). In paper [4], we gave some sufficient conditions for an integer $m$ to satisfy $3 \mid H(m)$ and $m \equiv 1(\bmod 4)$. In this paper, using the result of Uchida [5], we give moreover a sufficient condition for an integer $m$ to satisfy $4 \mid H(m)$ and $m \equiv 3(\bmod 4)$ (Proposition 3). Finally, we give numerical examples of some square-free integers $m$ satisfying $4 \mid H(m)$ and $m \equiv 3(\bmod 4)$.

The author would like to thank the referee for his valuable advices.

MaIN Theorem. Let $q$ be a prime and $k / Q$ be a real cyclic extension of degree $q$. If $m$ is the conductor of $k$, then the ideal class group $C(m)$ has a subgroup which is isomorphic to $C(k)^{q}$.

Received August 28, 1987. 
Proof. First, we prove this Theorem in the case of $q=2$. Let $k=$ $\boldsymbol{Q}(\sqrt{n})$ be a real quadratic field, where $n$ is a square-free integer. Let $m$ be the discriminant of $k$. Hence $m$ is the conductor of $k$. Now assume that $p_{1}, p_{2}, \cdots, p_{t}$ are all the prime divisors of $m$. Let $k^{*}$ be the genus field of $k$, that is, $k^{*}=\boldsymbol{Q}\left(\sqrt{p_{1}^{*}}, \sqrt{p_{2}^{*}}, \cdots, \sqrt{p_{t}^{*}}\right)$, where if $p$ is an odd prime, then $p^{*}=(-1)^{(p-1) / 2} p$, if $p=2$, then $p^{*}=-4,8$ or -8 according $n \equiv 3(\bmod 4), 2(\bmod 8)$ or $-2(\bmod 8)$ (see Ishida [1, Chapter 1]). Let $\tilde{k}$ be the Hilbert class-field of $k$ and $M=k^{*} \cap \tilde{k}$. Further let $H$ be a subgroup of the ideal class group $C(k)$ of $k$ and $H$ be isomorphic to the Galois group of $\tilde{k} / M$. From [1, Chapter 1], the Galois group of $k^{*} / k$ is isomorphic to $(Z / 2 Z)^{t-1}$. Hence $C(k)^{2}$ is a subgroup of $H$. On the other hand, since $M=k^{*} \cap \tilde{k}$, we can see that $M$ is contained in the real cyclotomic field $K=\boldsymbol{Q}\left(\zeta_{m}+\zeta_{m}^{-1}\right)$. Since $k^{*}$ is the genus field of $k$, we have $K \cap \tilde{k}=M$. Hence we have that $K \tilde{k} / K$ is an abelian unramified extension and the Galois group of $K \tilde{k} / K$ is isomorphic to the Galois group of $\tilde{k} / M$. Since the Galois group of $\tilde{k} / M$ is isomorphic to $H$ and $H$ has a subgroup $C(k)^{2}$, the Galois group of $K \tilde{k} / K$ has a subgroup which is isomorphic to $C(k)^{2}$. Hence the ideal class group $C(m)$ has a subgroup which is isomorphic to $C(k)^{2}$.

Next, we prove this Theorem in the case of an odd prime $q$. Let $k / \boldsymbol{Q}$ be a cyclic extension of degree $q$. Let $\tilde{k}$ be the Hilbert class field of $k$ and $k^{*}$ be the genus field of $k$. Further let $H$ be a subgroup of the ideal class group $C(k)$ of $k$ and $H$ be isomorphic to the Galois group of $\tilde{k} / k^{*}$. From [1, Theorem 5], we have that the Galois group of $k^{*} / k$ is isomorphic to $(Z / q Z)^{t-1}$, where $t$ is the number of distinct prime factors of the conductor $m$ of $k$. It is now easy to see that $C(k)^{q}$ is a subgruop of $H$. On the other hand, $k^{*}$ is contained in the real cyclotomic field $K=\boldsymbol{Q}\left(\zeta_{m}+\zeta_{m}^{-1}\right)$ (see Ishida [1, Theorem 5]). Since $k^{*}$ is contained in $\tilde{k}$ and $k^{*}$ is the genus field of $k$, we have $K \cap \tilde{k}=k^{*}$. In the same way as in the proof of this Theorem for the case $q=2$, we can show that the ideal class group $C(m)$ has a subgroup which is isomorphic to $C(k)^{q}$.

Remark. Let $n$ be a natural number. Let $h(k)$ be the class-number of $k$. If $n \mid h(k)$ and $q \nmid n$, then we have $n \mid H(m)$.

LEMMA 1. If an integer $m=A^{2 n}+4 B^{2 n}>5$ is square-free for natural numbers $n>1, A, B$, the ideal class group of a real quadratic field $\boldsymbol{Q}(\sqrt{m})$ has a cyclic subgroup with order $n$ (see Nakahara [2, Theorem 1]). 
Proposition 1. If an integer $m=A^{2 n}+A B^{2 n}>5$ is square-free for natural numbers $n>1, A, B$, then we have

(1) $n \mid H(m)$, if $n$ odd,

(2) $(n / 2) \mid H(m)$, if $n$ is even.

Proof. It is clear that $m \equiv 1(\bmod 4)$. Hence $m$ is the conductor of a real quadratic field $k=\boldsymbol{Q}(\sqrt{m})$. By Lemma 1, the ideal class group $C(k)$ of $k$ has a subgroup which is isomorphic to $Z / n Z$. Hence by Main Theorem, we have this Theorem.

LEMma 2. For any given natural number n, there exist infinitely many cubic cyclic fields $k$ whose ideal class groups contain a subgroup isomorphic to $(Z / n Z)^{2}$ (see Nakano [3, Theorem]).

Remark. Let $m$ be the conductors of $k$. From the proof of [3, Theorem], we have $3 \nmid m$, Hence $m$ are square-free integers.

By Lemma 2, we have

CoRollary. For any given natural number $n$, there exist infinitely many cubic cyclic fields $k$ whose ideal class groups $C(k)$ contain a subgroup isomorphic to $(Z / 3 n Z)^{2}$. Further the conductors $m$ of $k$ are squarefree integers.

Proposition 2. For any given natural number $n$, there exist infinitely many positive square-free integers $m$ such that the ideal class group $C(m)$ has a subgroup which is isomorphic to $(Z / n Z)^{2}$.

Proof. By Corollary of Lemma 2, there exist infinitely many cubic cyclic fields $k$ such that $C(k)^{3}$ has a subgroup which is isomorphic to $(Z / n Z)^{2}$ for any given natural number $n$. Let $m$ be the conductors of the cubic cyclic fields $k$. Hence $m$ are square-free integers. Then by Main Theorem, there exist infinitely many positive square-free integers $m$ such that the ideal class group $C(m)$ has a subgroup which is isomorphic to $(Z / n Z)^{2}$ for any given natural number $n$. This completes the proof.

Lemma 3. Let $q$ be a prime and $L / K$ be a cyclic extension of degree q. Let $C(L)$ and $C(K)$ be the ideal class groups of $L$ and $K$, respectively. Let $h(K)$ be the order of $C(K)$ and $p$ be a prime such that $p \nmid q h(K)$. Further let $f$ be the order of $p \bmod q$.

If $C(L)$ has a subgroup which is isomorphic to $Z / p^{r} Z$, then $C(L)$ has a subgroup which is isomorphic to $\left(Z / p^{r} Z\right)^{f}$ for some integer $r \geqq 1$ (see 
Washington [6, Theorem 10.8]).

Let $\ell$ be a prime. Let $q, q_{1}$ and $q_{2}$ be primes which satisfy the following conditions

(1) 2 or 3 is not an $\ell$-th power residue $\bmod q$ for $\ell=2$,

(2) 2 is not an $\ell$-th power residue $\bmod q_{i}(i=1,2)$ and 3 is an $\ell$-th power residue $\bmod q_{1}$ but is not an $\ell$-th power residue $\bmod q_{2}$ for an odd prime $\ell$.

Lemma 4. Let $n$ be a natural number. Let $m=\left(a^{2 n}+27\right) / 4$ for some integer a prime to 6. If a has prime factors $q, q_{1}$ and $q_{2}$ which satisfy the above conditions (1) and (2) for the prime factors $\ell$ of $n$, the ideal class group of the cubic cyclic field defined by

$$
f(x)=x^{3}+m x^{2}+2 m x+m=0
$$

has a subgroup which is isomorphic to $Z / n Z$ (see Uchida [5, Theorem 1]).

By Lemma 3 and Lemma 4, we have

CoRollary. Under the same assumptions as in Lemma 4, the ideal class group of the cubic cyclic field defined by

$$
f(x)=x^{3}+m x^{2}+2 m x+m=0
$$

has a subgroup which is isomorphic to $Z / n Z \oplus Z / n_{0} Z$, where $n_{0} \mid n$ and any prime factor of $n_{0}$ is congruent to $2(\bmod 3)$.

Proposition 3. Let a be an integer prime to 6, and assume that a has a prime factor $q$ such that $q \equiv \pm 5(\bmod 12)$ or $q \equiv \pm 11(\bmod 24)$.

If $m=\left(a^{4}+27\right) / 4$ is a sequare-free integer, then we see that $4 \mid H(m)$ and $m=3(\bmod 4)$.

Proof. It is easy to see that $m \equiv 3(\bmod 4)$. If $q \equiv \pm 11(\bmod 24)$, then we have $\left(\frac{2}{q}\right)=-1$. If $q \equiv 5(\bmod 12)$, then we have $\left(\frac{3}{q}\right)=-1$. Hence by Corollary, the ideal class group of the cubic cyclic field $k$ defined by

$$
f(x)=x^{3}+m x^{2}+2 m x+m=0
$$

has a subgroup which is isomorphic to $(Z / 2 Z)^{2}$. Since $m$ is a square-free integer, the discriminant of $k$ is equal to $m^{2}$ (see Uchida [5, Lemma 2]). Hence $m$ is the conductor of $k$. Therefore by Main Theorem, we have $4 \mid H(m)$. This completes the proof. 
Now we give some examples of square-f̂ree integers $m$ satisfying the conditions in Proposition 3 , that is, $4 \mid H(m)$ and $m \equiv 3(\bmod 4)$.

$163,607,19 \cdot 193,7 \cdot 1021,20887,32587,127 \cdot 769,7 \cdot 25261,373 \cdot 619,375163$, $103 \cdot 4549,7 \cdot 43 \cdot 2347,19 \cdot 75853,1972627,379 \cdot 7993,313 \cdot 11059,19 \cdot 349 \cdot 673$, $577 \cdot 8731,8788267,1789 \cdot 5443,7 \cdot 1694941,7 \cdot 31 \cdot 60139,3259 \cdot 4813,17143747$, $20362663,19 \cdot 1480933,32769907,35289547$.

\section{REFERENCES}

[1] Ishida, M., The genus fields of algebraic number fields, Lecture Notes in Math., 555, Berlin, Heidelberg, New York: Springer 1976.

[2] Nakahara, T., On real quadratic fields whose ideal class groups have a cyclic $p$ subgroup, Rep. Fac. Sci. Engin. Saga Univ., 6 (1978), 91-102.

[ 3 ] Nakano, S., Ideal class groups of cubic cyclic fields, Acta Arith., 46 (1986), 297-300.

[4] Osada, H., Note on the class-number of the maximal real subfield of a cyclotomic field, Manuscripta Math., 58 (1987), 215-227.

[ 5 ] Uchida, K., Class numbers of cubic cyclic fields, J. Math. Soc. Japan, 26 (1974), $447-453$.

[6] Washington, L., Introduction to cyclotomic fields, Graduate Texts in Math., 83, Berlin, Heidelberg, New York: Springer 1982.

Department of Mathematics

Rikkyo University

Ikebukuro, Tokyo 171, Japan 